\title{
Neumotórax espontáneo y enfisema bulloso bilateral
}

\author{
Spontaneous pneumothorax and bilateral bullous emphysema
}

\author{
Germán Brito-Sosa ${ }^{1} \mathbb{D}$, Ana María Iraizoz-Barrios² $\mathbb{D}$ \\ 1 Médico, especialista de primer grado en Cirugía General, especialista de primer grado en Medicina General Integral, máster de \\ Urgencias Médicas en Atención Primaria, Servicio de Cirugía General, Hospital Teófilo Dávila; investigador agregado de la Uni- \\ versidad Técnica de Machala, Machala, Ecuador. Miembro de la Sociedad Española de Cirugía Torácica \\ 2 Médica, especialista de primer grado en Laboratorio Clínico, especialista de primer grado en Medicina General Integral; profesora \\ e investigadora, Universidad Técnica de Machala, Machala, Ecuador.
}

\section{Resumen}

Introducción. El objetivo de este artículo fue reportar el caso de un paciente con antecedente de enfisema bulloso bilateral que le ocasionó un neumotórax persistente de difícil tratamiento.

Caso clínico. Se trata de un paciente de 50 años de edad con diagnóstico de neumotórax asociado con un enfisema bulloso bilateral, en quien estaba contraindicado el tratamiento quirúrgico. El cuadro clínico comenzó con dolor en el hemitórax derecho y disnea, que se fue intensificando hasta el punto de no tolerar el decúbito. En la radiografía de tórax se observó colapso del pulmón derecho con múltiples bulas en ambos pulmones, por lo que se practicó una pleurotomía mínima alta derecha. La evolución del paciente no fue favorable por persistir el neumotórax, lo cual llevó al uso de un tratamiento alternativo.

Discusión. En los pacientes con neumotórax persistente por enfisema bulloso bilateral sin indicación quirúrgica, el talco es una alternativa menos invasiva para lograr una sínfisis pleural con buenos resultados, método cuya utilidad se demuestra.

Palabras clave: neumotórax; enfisema pulmonar; enfermedades pulmonares; diagnóstico por imagen; cirugía torácica asistida por video; talco.

\footnotetext{
Abstract

Introduction. The aim of this report was to present the case of a patient with a history of bilateral bullous emphysema that causes a persistent pneumothorax that was difficult to treat.

Clinical case. This is a 50-year-old patient diagnosed with pneumothorax associated with bilateral bullous emphysema, in whom surgical treatment being contraindicated. The clinical picture began with pain in the right hemithorax and dyspnea, which intensified to the point of not tolerating decubitus. In the chest X-ray, collapse of

Fecha de recibido: 21/07/2019 - Fecha de aceptación: 28/01/2020

Correspondencia: Germán Brito-Sosa, Callejón 6ts sur entre 14ava Oeste y 15ava Oeste, Ciudadela 9 de Octubre, Ciudad Machala, Provincia El Oro, Ecuador. Teléfono: 0053959289364. Correo electrónico: german.brito512@yahoo.es

Citar como: Brito-Sosa G, Iraizoz-Barrios AM. Neumotórax espontáneo y enfisema bulloso bilateral. Rev Colomb Cir. 2021;36:155-60. https://doi.org/10.30944/20117582.122

Este es un artículo de acceso abierto bajo una Licencia Creative Commons - BY-NC-ND https://creativecommons.org/licenses/by-ncnd/4.0/deed.es
} 
the right lung was observed with multiple bullae in both lungs, therefore a right upper minimum pleurotomy was performed. The patient's evolution was not favorable due to persisting pneumothorax, which led to the use of an alternative treatment.

Discussion. In patients with persistent pneumothorax due to bilateral bullous emphysema without surgical indication, talc is a less invasive alternative to achieve a pleural symphysis with good results, a method whose utility is demonstrated.

Keywords: pneumothorax; pulmonary emphysema; lung diseases; diagnostic imaging; thoracic surgery, videoassisted; talc.

\section{Introducción}

El neumotórax es una entidad clínica caracterizada por la existencia de aire en el espacio interpleural, entre la pleura visceral y la parietal, que ocasiona colapso pulmonar ${ }^{1-3}$. Como lo relatan algunos autores, fue el médico francés Itard, en 1803, quien introdujo en la práctica médica el término neumotórax para nombrar la presencia no explicada de aire en la cavidad pleural ${ }^{4,5}$.

Una de las alteraciones pulmonares que puede ocasionar un neumotórax es el enfisema pulmonar, la cual es una enfermedad crónica e invalidante que afecta del 10 al $15 \%$ de la población mundial ${ }^{6}$. Desde el punto de vista fisiopatológico, se describe como la destrucción de las paredes alveolares sin fibrosis obvia, lo que produce dilatación de los espacios aéreos distales al bronquiolo terminal y la formación de pequeñas y grandes bullas (burbujas); su principal causa es el tabaquismo ${ }^{7}$.

Existen pocos reportes en la literatura científica de pacientes con neumotórax persistente por enfisema bulloso bilateral sin indicación quirúrgica, por lo que se decidió presentar el caso y mostrar que se dispone de alternativas menos invasivas para este tipo de paciente.

\section{Reporte de caso}

Se trata de un paciente de 50 años de edad, con antecedentes de padecer asma bronquial y ser fumador de dos cajetillas diarias de cigarros desde 30 años antes, lo cual le había provocado enfisema bulloso bilateral. Acudió al servicio de urgencias después de veinticuatro horas de presentar ligero dolor en el hemitórax derecho y disnea, la cual se fue intensificando hasta el punto de no tolerar el decúbito.

Como datos positivos en el examen físico del aparato respiratorio, se encontró tiraje supraclavicular e intercostal, con disminución de la expansión torácica y abombamiento del hemitórax derecho; a la palpación, las vibraciones vocales estaban disminuidas; a la percusión, había hipersonoridad y, a la auscultación, el murmullo vesicular estaba ausente.

En la radiografía postero-anterior inicial de tórax en bipedestación se encontró colapso del pulmón derecho con múltiples bullas enfisematosas en ambos campos pulmonares (figura 1).

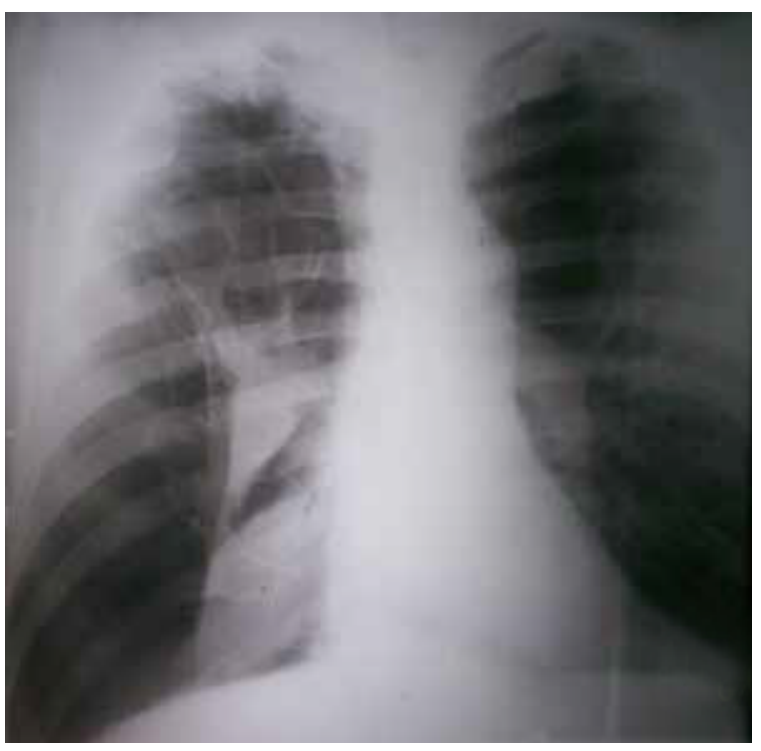

Figura 1. Radiografía de tórax, postero-anterior y en bipedestación, en la que se observa el colapso del pulmón derecho con múltiples bullas enfisematosas en ambos campos pulmonares. 
El lóbulo superior derecho presentaba signos de atrapamiento de aire por la existencia de enfisema pulmonar, con aumento de su radiotransparencia. Las costillas se encontraban 'horizontalizadas' y había ensanchamiento de los espacios intercostales, y descenso y aplanamiento del diafragma.

Con la sintomatología del paciente y los hallazgos radiológicos, se diagnosticó neumotórax espontáneo secundario a enfisema. Se decidió practicar una pleurotomía mínima alta derecha. La evolución del paciente no fue favorable por persistir el neumotórax, además de aparecer un proceso infeccioso pulmonar a los cuatro días, lo cual ocasionó empiema pleural e incremento de la disnea.

En el leucograma del momento del derrame pleural se encontró: leucocitosis con predominio de los polimorfonucleares (leucocitos: 12,6 x $10^{9} / \mathrm{L}$; polimorfonucleares: 85,9\%). Se decidió practicar una pleurotomía mínima baja, añadiendo otro antibiótico y manteniendo la terapia respiratoria, medidas que contribuyeron a la mejoría de la sintomatología. Posteriormente, al pinzar los drenajes torácicos, el pulmón se colapsó nuevamente, por lo que se le hizo una tomografía computarizada (TC). Se observó escaso parénquima pulmonar con múltiples bullas de diferente tamaño diseminadas en ambos campos pulmones, algunas de ellas con niveles hidroaéreos en su interior en la base del hemitórax derecho; también, había moderado colapso pulmonar derecho (figura 2).

Se retiró la sonda torácica obstruida y se practicó una nueva pleurotomía mínima baja, manteniéndose con una aspiración negativa a $-20 \mathrm{~cm}$ de agua. El derrame disminuyó considerablemente, pero, al suspender la aspiración negativa, el pulmón colapsó nuevamente. El paciente se negaba a otros procedimientos quirúrgicos, por lo que, mediante consulta con el grupo de especialistas, se decidió optar por una pleurodesis con talco por la sonda, después de la cual, se logró mantener expandido el pulmón (figura 3).

\section{Discusión}

Según su etiología, un neumotórax puede clasificarse como espontáneo, traumático o iatrogénico.

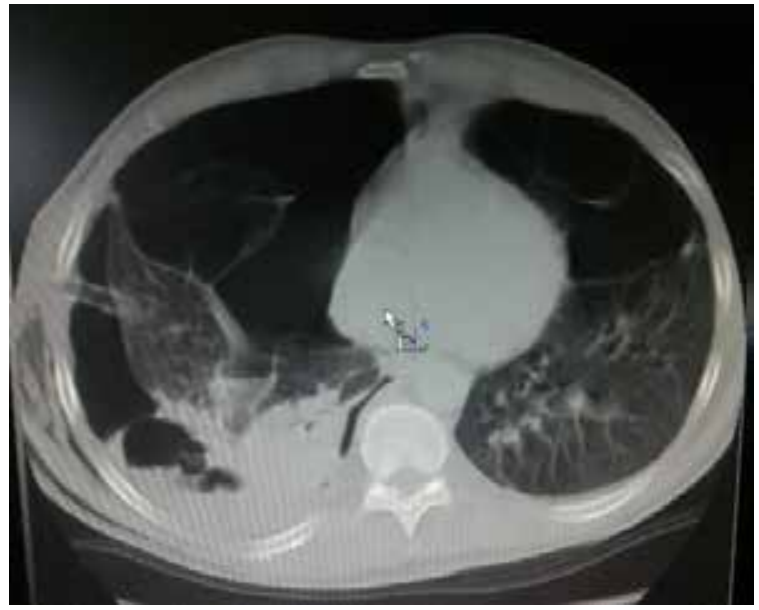

Figura 2. Tomografía computarizada de tórax en la que se observa escaso parénquima pulmonar, con múltiples bullas de diferentes tamaños localizadas en ambos campos pulmones, algunas de ellas con niveles hidroaéreos en su interior en la base del hemitórax derecho. También, se observa moderado colapso pulmonar derecho.

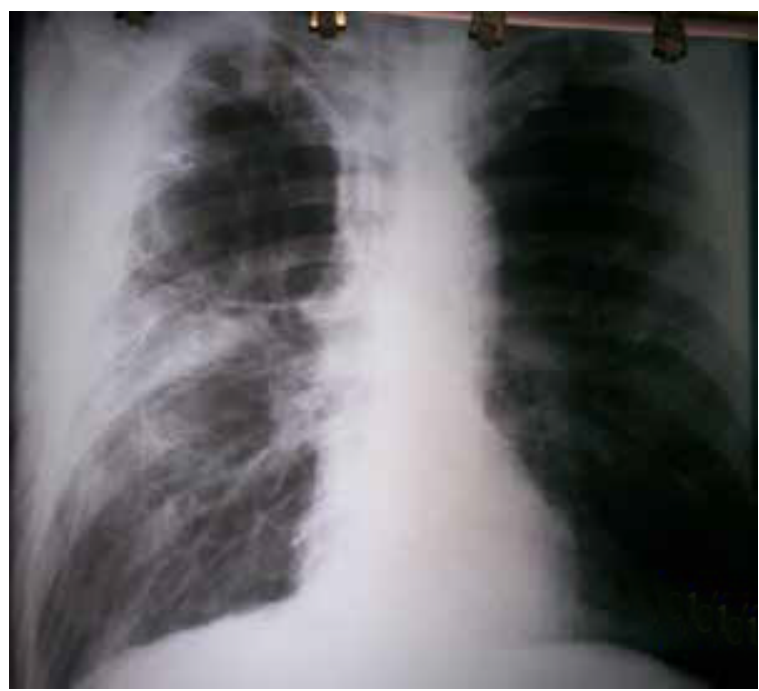

Figura 3. Radiografía de tórax, postero-anterior y en bipedestación, en la que se aprecia la reexpansión del pulmón derecho, además de múltiples bullas enfisematosas en ambos campos pulmonares.

El neumotórax espontáneo aparece sin ningún traumatismo previo y se puede subclasificar como primario, cuando no hay enfermedad pulmonar subyacente, o como secundario, cuando síla hay ${ }^{8}$. 
El neumotórax espontáneo se observa con mayor frecuencia en el hombre, con una relación de 6 a 1 , como resultado de pequeños esfuerzos, una crisis de tos, etc. Puede ser derecho o izquierdo, aunque la mayor parte de los autores coinciden en que es más frecuente en el hemitórax derecho ${ }^{7}$.

La mayoría de los casos de neumotórax espontáneo primario se presentan en hombres entre los 10 y los 30 años de edad, y rara vez en mayores de 40 años, aparentemente saludables, altos y delgados, y se atribuyen a la formación y posterior ruptura de ampollas subpleurales congénitas en los vértices pulmonares. El neumotórax espontáneo secundario aparece como una complicación de alguna afección pulmonar ya existente ${ }^{9,10}$, como enfisema pulmonar, asma bronquial, fibrosis quística, neoplasia, tuberculosis pulmonar, procesos pulmonares infecciosos o endometriosis pulmonar ${ }^{8,11}$.

Por su acción destructora sobre el parénquima pulmonar, el tabaquismo es el principal factor de riesgo, pues incrementa hasta en 20 veces el riesgo de desarrollar un neumotórax ${ }^{9}$. Un neumotórax se considera persistente si la fuga de aire dura más de cinco días ${ }^{12}$.

La bulla gigante es una entidad rara que ocurre típicamente asociada con el enfisema ${ }^{13}$. Burke describió el enfisema bulloso gigante por primera vez en 1937. Esta enfermedad es característica en hombres jóvenes, delgados y fumadores. Para ser considerada gigante, una bulla debe ocupar, al menos, un tercio del hemitórax ${ }^{14}$. Suele ser unilateral y limitada a los ápices. Por el contrario, el enfisema bulloso produce afectación bilateral ${ }^{15}$, como se pudo apreciar en el presente paciente.

El diagnóstico de neumotórax espontáneo secundario a enfisema bulloso bilateral se logra con una anamnesis detallada y una correcta exploración física ${ }^{16}$. La radiografía de tórax es la prueba de elección por su disponibilidad y bajo costo, y es fundamental para conocer el volumen del neumotórax y las lesiones asociadas.

La tomografía computarizada (TC) no se recomienda de forma rutinaria en todo paciente con un primer episodio de neumotórax espontáneo. No obstante, actualmente se considera el método diagnóstico estándar en caso de neumotórax es- pontáneo cuando está asociado a otras enfermedades pulmonares subyacentes, en los pacientes con episodios recurrentes, para estimar el tamaño real del neumotórax, y para confirmar o descartar una mala posición del drenaje torácico. La TC presenta sensibilidad y especificidad cercanas al $100 \%$; además, brinda información más detallada, como el número y la localización de las bullas, ipsilaterales o contralaterales, o sobre enfermedades pulmonares subyacentes ${ }^{9}$. Es valioso medir la concentración sérica de la alfa ${ }_{1}$-antitripsina para confirmar la causa del enfisema bulloso bilateral; la deficiencia de esta proteína indica una enfermedad enfisematosa de origen congénito ${ }^{17}$.

En casos de neumotórax recurrente o persistente, cuando el pulmón permanece sin expandir a pesar del correcto funcionamiento del drenaje torácico, el tratamiento más aceptado es el quirúrgico. Mediante videotoracoscopia y con endograpadoras, se resecan las ampollas subpleurales y, posteriormente, se procede a la abrasión pleural y la pleurodesis química para lograr la sínfisis pleural ${ }^{3,18}$. Las posibilidades de recidiva durante el primer episodio son mayores en los pacientes con neumotórax secundario a enfermedad pulmonar obstructiva crónica, pero las recurrencias se reducen al $25 \%$ con la pleurodesis.

En el presente paciente, se presentó como complicación un empiema pleural, cuya causa más frecuente es un proceso neumónico y que acompaña entre el 60 y el $70 \%$ de todos los casos de neumonía. Otras causas menos frecuentes de empiema pleural son las quirúrgicas, la tuberculosis, los traumas y las neoplasias.

El tratamiento quirúrgico se encaminó a tratar la infección. Las distintas alternativas quirúrgicas para tratar el empiema pleural van desde la pleurotomía hasta la decorticación por toracotomía, según la fase en que se encuentre el empiema pleural: fase I o exudativa, fase II o fibrinopurulenta, o fase III de organización o consolidación. Los procedimientos mínimamente invasivos, como la cirugía videotoracoscópica o video-asistida, son la vía de abordaje más utilizada en los empiemas pleurales cuando se diagnostican por primera vez. En este paciente, se resolvió con la pleurotomía, procedimiento que se considera de elección para 
el empiema pleural en sus fases iniciales, cuando existen enfermedades asociadas que contraindiquen la cirugía y cuando la expectativa de vida es limitada ${ }^{18}$.

En múltiples estudios se ha demostrado la superioridad del talco (91\% de eficacia) para la pleurodesis, comparado con la bleomicina y la doxiciclina ${ }^{19}$. En la práctica, el talco es uno de los productos más utilizados para la pleurodesis, en los países occidentales y en Japón ${ }^{2}$. El talco, polvo hidratado de silicato de magnesio, es extremadamente efectivo y el agente más barato para inducir la formación de adherencias pleurales ${ }^{3,10}$. Promueve la llegada de células polimorfonucleares y, posteriormente, de macrófagos a la pleura, e incrementa la producción de interleucina 8 (IL-8) y de factor quimiotáctico de monocitos 1 (MCP-1); de esta manera, produce una pleuritis que alcanza su máximo efecto entre las 3 y las 24 horas ${ }^{10}$.

La pleurodesis evita los riesgos de la cirugía y es útil en pacientes de edad avanzada; además, es una buena opción cuando la cirugía está contraindicada o el paciente la rechaza. Por consiguiente, este método es una buena alternativa que debemos tener siempre presente y cuyo objetivo es mejorar la calidad de vida ${ }^{20}$.

Existen dos formas de administrar el talco. Una es mediante la toracoscopia y la otra consiste en instilar por el drenaje pleural $5 \mathrm{~g}$ de talco disuelto en 20 a $30 \mathrm{ml}$ de solución salina al 0,9\%, cerrar el drenaje durante una hora, aconsejar al paciente que realice movimientos de rotación y reabrir el drenaje conectado a una aspiración de $-20 \mathrm{~cm}$ de agua. El uso de talco tiene sus complicaciones, como son: fiebre, empiema, dolor torácico de intensidad variable y dificultad respiratoria del adulto. Afortunadamente, esta última es poco habitual y se ha atribuido a dosis excesivas de talco $^{11,19}$. Inicialmente, existieron informes sobre el efecto cancerígeno del talco, los cuales se originaron probablemente por el contenido de impurezas como el amianto, y son especulaciones y no hechos demostrados ${ }^{21}$.

La tasa de mortalidad por neumotórax espontáneo primario se estima en 0,09\% en hombres y $0,06 \%$ en mujeres; para la forma secundaria es mayor, debido a la enfermedad pulmonar de base y a una menor reserva funcional respiratoria ${ }^{9}$. En los pacientes con enfisema pulmonar con déficit de alfa ${ }_{1}$-antitripsina, en general, los resultados del tratamiento médico y el quirúrgico no son satisfactorios y el trasplante pulmonar sería la única opción posible ${ }^{17}$.

\section{Conclusión}

No siempre el neumotórax espontáneo causado por un enfisema bulloso es de fácil tratamiento y este paciente es un ejemplo de lo difícil que puede ser lograr la reexpansión pulmonar. Debido al gran riesgo quirúrgico, al final, se logró con la pleurodesis con talco, método este que puede ser una alternativa en casos seleccionados de alto riesgo.

\section{Cumplimiento de normas éticas}

Consentimiento informado. Este estudio es una presentación de caso con revisión retrospectiva de la historia clínica y se obtuvo el consentimiento informado del paciente.

Declaración de conflicto de intereses. Los autores declaran no tener ningún conflicto de intereses.

Fuentes de financiación. Recursos propios de los autores.

Contribución de los autores: Concepción y diseño del estudio, adquisición de datos, redacción del manuscrito: Germán Brito-Sosa.

Redacción del manuscrito y revisión crítica: Ana María Iraizoz-Barrios.

\section{Referencias}

1. García A, Pardo G, del Cueto H. Neumotórax. Traumatismos del tórax. En: Cruz L, editor. Texto de Cirugía. La Habana: Editorial Ciencias Médicas; 2010. p. 282-5.

2. Hamada S, Okamoto N, Watanabey I, Tsukino M. ¿Es segura la pleurodesis con solución de glucosa al 50\% en pacientes con neumotórax espontáneo? A propósito de una serie de casos. Arch Bronconeumol. 2016;53:210-11. https://doi.org/10.1016/j.arbres.2016.08.018

3. Berkel V, Kuo E, Meyers BF. Pneumothorax, bullous disease, and emphysema. Surg Clin North Am. 2010;90:935-53.

https://doi.org/10.1016/j.suc.2010.06.008 
4. Armas JC, Valdés PG, Rodríguez A, Valdés FR. Algunos aspectos clinicoquirúrgicos del neumotórax espontáneo. Rev Cubana Cir. 2001;40:7-11.

5. DeVries WC, Wolfe WG. The Management of Spontaneous Pneumothorax and Bullous Emphysema. Surg Clin NA. 1980;60:851-66. https://doi.org/10.1016/S0039-6109(16)42187-0

6. Leal A, Llorens JA, Ramírez ET, Mederos ON, Adefna RI. Reducción simultánea del enfisema bulloso bilateral por esternotomía media. Rev Cubana Cir. 2000;39:195-203.

7. Roca R, Smith VV, Paz E, Lasada J, Serret B, Llamos N, et al. Enfermedad pulmonar obstructiva crónica. Enfermedades del sistema respiratorio. Temas de Medicina Interna. 4a ed. Robert Z, Hechevarria G, editores. La Habana: Editorial Ciencias Médica. 2002. p. 87-112.

8. Brito G, Vivo JG. Incidencia del neumotórax en el Hospital "Julio Trigo López". Rev Cubana Cir. 2012;5:10-6.

9. Aguinagalde B, Aranda JL, Busca P, Martínez I, Royo I, Zabaleta J. Guía de práctica clínica de la SECT sobre el manejo de pacientes con neumotórax espontáneos. Rev Cir Esp. 2017;96:3-11. https://doi.org/10.1016/j.ciresp.2017.11.005

10. Guelbenzu JJ, Vila E, Ágreda J. El neumotórax espontáneo: revisión de 130 casos. An Sist Sanit Navar. 2001;24:307-13.

11. Velásquez ME, Velásquez M. Neumotórax catamenial. Rev Colomb Cir. 2015;30:286-91.

12. Navarro A, Pastor MD, León MC, Reyes SB, Fuster JL. Pleurodesis con sangre autóloga: una solución, segura y eficaz para el tratamiento de neumotórax persistente. Rev Pediatr (Barcelona). 2016;85:117-66. https://doi.org/10.1016/j.anpedi.2015.11.010

13. González P, Hernández JM, Fumero S. Evolución de una bulla gigante. J Med Gen Fam. 2018;7:66-9. https://doi.org/10.24038/mgyf.2018.017
14. Ramadas P, Chakravarty R, Krishnan P, Nadkarni A. Dangers of flying high and diving low! An unusual case of dyspnea. Respir Med Case Rep. 2016;20:1-3. https://doi.org/10.1016/j.rmcr.2016.10.015

15. Yunhee I, Farooqi s, Mora A. Vanishing lung syndrome. Proc (Bayl Univ Med Cent). 2016;29:399-401. https://doi.org/10.1080/08998280.2016.11929486

16. Reyes FA, Pérez ML, Figueredo EA, Céspedes Y, Fernández A. El método clínico aplicado al diagnóstico del dolor torácico agudo. Correo Científico Médico (CCM). 2018;22:474-95.

17. Leal A, Ramos N, Castellanos JA, Adefna RI, Riano MA, González AJ. Enfisema bulloso bilateral gigante por déficit de alfa1-antitripsina. Rev Cubana Cir. 2009;48:1-4.

18. González R, Prats R, Lazo D, Jadue A, Mordojovich G, Santolaya R, et al. Empiema pleural en 343 casos con tratamiento quirúrgico: características, resultados inmediatos y factores asociados a morbilidad y mortalidad. Rev Chil Cir. 2012;64:32-39. https://doi.org/10.4067/S0718-40262012000100006

19. Izquierdo-Elena I. Pleurodesis. Kirurgia: Universidad del País Vasco. España; 2006. Fecha de consulta: 15 de diciembre de 2018. Disponible en:

http://www.sc.ehu.es/scrwwwsr/kirurgia/20066/ pleurodesis.htm

20. Fuentes EA, Rivera JM, Canales ZJ, Ávalos TM, Alarcón CE, González LR. Caracterización y resultados de la pleurodesis con talco en derrame pleural neoplásico. ANACEM. 2015;9:62-7.

21. Mármol EE, Martínez S, Baldó X, Rubio M, Penagos JC, Quetglás FS. Eficacia y morbimortalidad del tratamiento quirúrgico mediante videotoracoscopia y pleurodesis con talco del neumotórax espontáneo primario. Cir Esp. 2011;89:463-67. https://doi.org/10.1016/j.ciresp.2011.02.016 